\title{
Serendipitous Detection of Asymptomatic Lesions in International Travellers
}

\author{
William Wen $\mathrm{Oh}^{1}$, Gerard Thomas Flaherty ${ }^{1,2^{*}}$ \\ ${ }^{1}$ School of Medicine, National University of Ireland Galway, Galway, Ireland \\ ${ }^{2}$ School of Medicine, International Medical University, Kuala Lumpur, Malaysia
}

Corresponding Author: Gerard Thomas Flaherty, Professor, School of Medicine, National University of Ireland Galway, Galway, Ireland. Tel: +353-91495469, Email: gerard.flaherty@nuigalway.ie

Received September 27, 2019; Accepted November 12, 2019; Online Published December 15, 2019

Citation: Oh WW, Flaherty GT. Serendipitous detection of asymptomatic lesions in international travellers. Int J Travel Med Glob Health. 2019;7(4):147-148. doi:10.15171/ijtmgh.2019.30.

\section{Dear Editor,}

The recent case of a 41-year-old British tourist, whose breast cancer was first suggested by a visit to a popular tourist attraction in Scotland, prompted us to consider how travel may serendipitously yield unforeseen diagnoses. The woman and her family were entertained in the thermal imaging camera room of the Camera Obscura and World of Illusions exhibit in Edinburgh when she noticed that her left breast had a different colour to her right breast. ${ }^{1}$ She recorded an image of the heat patch on her phone which she subsequently discussed with her doctor. A diagnosis of early-stage breast cancer was made and the patient was successfully treated with mastectomy without the need for chemo-radiotherapy. ${ }^{1}$ The manager of the facility was previously unaware of the potential for their thermal camera to detect occult cancers.

Thermal imaging is used at some international airports for the mass screening of passengers entering a jurisdiction, in order to detect the presence of fever, which appears as an area of intense thermal activity on the heat map. While this technology was used with some success during the 2002-2003 SARS outbreak, it has been criticised in a recent review, which questioned its effectiveness in the surveillance of groups of passengers in transit. ${ }^{2}$ To the best of our knowledge, no case of breast cancer has been reported in the context of airport thermal scanning.

With the intensification of airport security measures following the September 11, 2001 terrorist attacks in the United States, most airports employ scanners to detect concealed weapons and explosives in airline passengers. Since June 2013, the earlier full-body backscatter x-ray devices have been replaced in larger US airports by radiofrequency millimetre-wave scanners, which produce a mannequin-like image, with a yellow box to denote areas requiring a manual search by security personnel.

Accidental detection of skin lesions has occurred at airports using the newer generation of full-body scanners. Heymann reported the case of a traveller with a $1.5-\mathrm{cm}$ epidermal inclusion cyst, which had been revealed by a pat-down search of an area identified by the scanning device as being suspicious for hidden explosives. ${ }^{3}$ Mayer and Adams describe the case of a $1.35-\mathrm{mm}$ thick nodular melanoma, which was found on inspection of the left lower leg of a 50-year-old frequent male business traveller. The traveller concerned underwent repeated pat-down searches of this anatomical site on the basis of images displayed when posing within a fullbody scanner. Since he was successfully treated, he has had no further intrusive searches at airport security. ${ }^{4}$ A 68-year-old man opted to undergo definitive surgical management of an asymptomatic left inguinoscrotal hernia, rather than endure embarrassing examinations of his genital area precipitated by body scanner detections at US airports. ${ }^{5}$

The exquisite sensitivity of modern airport security equipment has raised novel diagnostic conundra which are of interest to the travel medicine community. Air travellers should be aware of the potential for both security scanners and thermal fever detection cameras at airports to disclose previously undetected lesions. Likewise, airport screeners should be familiar with this phenomenon in passengers and should follow a sensitive protocol in informing travellers that an abnormality may require additional follow-up with a doctor. Frequent travellers without known implants, who must submit to repetitive manual body searches as a result of scanning, should bring this matter to the attention of their physicians in order to rule out the presence of an occult cancer. Where a skin lesion is benign and does not warrant excision, the traveller should be advised to present a medical

Copyright $\odot 2019$ The Author(s). This is an open-access article distributed under the terms of the Creative Commons Attribution License (http:// creativecommons.org/licenses/by/4.0), which permits unrestricted use, distribution, and reproduction in any medium, provided the original work is properly cited. 
letter at airport security stations on future trips. It may be worthwhile to discuss this issue with air travellers during the pre-travel consultation.

\section{Authors' Contributions}

GTF led the conception of the manuscript. WWO and GTF contributed equally to the preparation of the first draft. Both authors read and approved the final version of the manuscript.

\section{Conflict of Interest Disclosures}

The authors declare that they have no conflicts of interest.

\section{Ethical Approval}

Not applicable.

\section{Funding/Support}

None.

\section{References}

1. British Broadcasting Corporation (BBC). Breast cancer detected by thermal imaging scan in Edinburgh. BBC; 2019. https://www.bbc. com/news/uk-scotland-edinburgh-east-fife-50139540. Accessed November 27, 2019.

2. Ring EF, Ammer K. Infrared thermal imaging in medicine. Physiol Meas. 2012;33(3):R33-46. doi:10.1088/0967-3334/33/3/r33.

3. Heymann WR. A Cyst Misinterpreted on Airport Scan as Security Threat. JAMA Dermatol. 2016;152(12):1388. doi:10.1001/ jamadermatol.2016.3329.

4. Mayer JE, Adams BB. Nodular melanoma serendipitously detected by airport full body scanners. Dermatology. 2015;230(1):16-17. doi:10.1159/000368045.

5. Naraynsingh V, Cawich SO, Maharaj R, Dan D. Inguinal hernia and airport scanners: an emerging indication for repair? Case Rep Med. 2013;2013:952835. doi:10.1155/2013/952835. 